\title{
Arsenene and Antimonene: Two-Dimensional Materials with High Thermoelectric Figures of Merit
}

\author{
S. Sharma, S. Kumar, and U. Schwingenschlögl \\ Physical Science and Engineering Division (PSE), King Abdullah University of Science \\ and Technology (KAUST), Thuwal 23955-6900, Saudi Arabia
}

(Received 8 April 2017; revised manuscript received 22 June 2017; published 25 October 2017)

\begin{abstract}
We study the thermoelectric properties of As and Sb monolayers (arsenene and antimonene) using density-functional theory and the semiclassical Boltzmann transport approach. The materials show large band gaps combined with low lattice thermal conductivities. Specifically, the small phonon frequencies and group velocities of antimonene lead to an excellent thermoelectric response at room temperature. We show that $n$-type doping enhances the figure of merit.
\end{abstract}

DOI: 10.1103/PhysRevApplied.8.044013

\section{INTRODUCTION}

To cope with growing energy demands, alternative approaches are required that can reduce the dependence on fossil fuels and other conventional energy resources. One important area is the conversion of waste heat into electricity with the help of thermoelectric devices [1-3]. The efficiency of the thermoelectric materials used in such devices is measured by a dimensionless quantity known as the figure of merit, $Z T=\left(S^{2} \sigma T\right) /\left(\kappa_{e}+\kappa_{l}\right)$, where $S$ is the Seebeck coefficient, $\sigma$ is the electrical conductivity, $\kappa_{e}$ is the electronic contribution to the thermal conductivity, and $\kappa_{l}$ is the lattice thermal conductivity. Since a high $Z T$ value is required at the mean operation temperature of a heat source, recent research focuses either on finding alternative thermoelectric materials or on adopting specific strategies for enhancing the $Z T$ value of known materials. The idea of reducing the dimension of a material has turned out to be particularly successful [4-6]. Therefore, it is not surprising that the thermoelectric properties of two-dimensional materials, including graphene [7], silicene [8,9], germanene [8], phosphorene [10-12], graphdiyne [13], transition-metal dichalcogenides [14,15], and MXenes [16], now attract a lot of attention.

Only recently, monolayers of arsenic (arsenene, As) and antimony (antimonene, $\mathrm{Sb}$ ) were predicted to exist and to be semiconducting, in contrast to few-layer structures $[17,18]$. While both buckled and puckered honeycomb structures are found to be stable [19], buckled arsenene is energetically favored over puckered arsenene [18]. Experimentally the possibility of growing antimonene has been explored on $\mathrm{Bi}_{2} \mathrm{Te}_{3}$ and $\mathrm{Sb}_{2} \mathrm{Te}_{3}$ substrates [20]. A multilayer arsenene nanoribbon was synthesized on an InAs substrate using a plasma-assisted process [21]. Since gray arsenic has a layered buckled structure [22], the exfoliation technique can be used to obtain arsenene similar

*udo.schwingenschlogl@kaust.edu.sa to other two-dimensional materials, such as graphene and phosphorene.

A series of studies has explored the electronic properties of arsenene and antimonene [23-32]. Thermal transport, on the other hand, was addressed in Ref. [33] for puckered arsenene by first-principles calculations and Boltzmann transport theory, demonstrating high anisotropy. The same approach applied to buckled antimonene yields, at $300 \mathrm{~K}$, a low lattice thermal conductivity of $15.1 \mathrm{~W} \mathrm{~m}^{-1} \mathrm{~K}^{-1}$ [34], which can be further reduced by chemical functionalization [35]. The buckled and puckered structures of antimonene were also studied in Ref. [36], and systematic insight into the interrelation between buckling and transport in two-dimensional group-IV and group-V materials was given in Ref. [37]. However, the thermoelectric properties of arsenene and antimonene have escaped attention. Both materials have large band gaps and their bulk counterparts are known for low thermal conductivities, so that they appear to be good candidates for thermoelectric purposes.

Various examples of thermoelectric applications of twodimensional materials have been reported in the literature in recent years. Graphene (monolayer [38,39] and bilayer [40]) exfoliated on a $\mathrm{SiO}_{2} / \mathrm{Si}$ substrate, for example, provides a significant thermoelectric response and the usual temperature dependence of $S$ can be overcome on a SiC substrate [41]. Performance improvements have been achieved through $\mathrm{O}$ plasma treatment [42] and molecular decoration [43]. Interestingly, high-density defects can also have positive effects [44]. Monolayer transition-metal dichalcogenides, such as $\mathrm{MoS}_{2}\left(S \sim 30 \mathrm{mV} \mathrm{K}^{-1}\right.$ at room temperature) [45] and $\mathrm{WSe}_{2}$ [46], provide higher values of $S$ but less mechanical stability. In this context, it is the aim of the present study to establish comprehensive insight into the potential of buckled arsenene and antimonene for thermoelectric applications, using first-principles calculations and Boltzmann transport theory. We also investigate the properties under $n$ - and $p$-type doping. 


\section{THEORETICAL APPROACH}

We use density-functional theory as implemented in the Vienna ab initio simulation package [47] with a 550-eV planewave cutoff energy. The unit cell consists of the twodimensional material complemented by a vacuum slab of $15-\AA$ thickness, in order to avoid an interaction between periodic images perpendicular to the monolayer. The generalized-gradient approximation of the exchange-correlation functional in the form of Perdew, Burke, and Ernzerhof is employed for fully relaxing the structures, including the unitcell parameters [48]. For the integration of the Brillouin zone, a $11 \times 11 \times 1 k$ mesh (Monkhorst-Pack scheme) is employed.

We solve the semiclassical Boltzmann transport equation in the constant relaxation-time approximation, using the BoltzTraP code [49]. To this aim, Kohn-Sham eigenvalues are obtained by the Heyd-Scuseria-Ernzerhof (HSE06) hybrid exchange-correlation functional [50], in order to accurately describe the band structure, and Fourier interpolated on a refined $22 \times 22 \times 1 k$ mesh. Both electron and hole transport are treated in the rigid-band approximation. A $41 \times 41 \times 1 k$ mesh is employed for calculating the density of states (DOS). With the second- and third-order force constants as input, $\kappa_{l}$ can be calculated using the SHENGBTE code [51,52]. No drawbacks are expected from the fact that phonon drag is not considered in this approach, because, at the elevated temperatures relevant for most thermoelectric applications, the contributions to $S$ are negligible [53]. The second-order force constants are determined by PHONOPY [54] with a $5 \times 5 \times 1$ supercell and a $4 \times 4 \times 1 k$ mesh. The same supercell with an interaction range of $7.7 \AA$ is used to obtain the third-order force constants, where symmetry analysis by SPGLIB [55] allows us to reduce the number of structures to be considered to 156 . For calculating phonon lifetimes, we employ a $100 \times 100 \times 1 q$ mesh. The lattice thermal conductivity is given by

$$
\kappa_{l}^{\alpha \beta}=\frac{1}{k_{B} T^{2} \Omega N} \sum_{\lambda} f_{0}\left(f_{0}+1\right)\left(\hbar \omega_{\lambda}\right)^{2} v_{\lambda}^{\alpha} F_{\lambda}^{\beta},
$$

where $k_{B}$ is the Boltzmann constant, $T$ the temperature, $\Omega$ the volume of the unit cell, $N$ the number of points of the $q$ mesh, $f_{0}$ the equilibrium phonon distribution function, $\hbar$ the reduced Planck constant, $\omega_{\lambda}$ the angular frequency of phonon mode $\lambda$ (comprising the phonon branch index and wave number), $\mathbf{v}_{\lambda}$ the group velocity, and $\mathbf{F}_{\lambda}$ the mean free displacement. $\mathbf{F}_{\lambda}=\tau_{\lambda}^{0}\left(\mathbf{v}_{\lambda}+\boldsymbol{\Delta}_{\lambda}\right)$ is solved iteratively starting from $\boldsymbol{\Delta}_{\lambda}=0$, where $\tau_{\lambda}^{0}$ represents the initial scattering time, until the change in $\kappa_{l}^{\alpha \beta}$ is less than $10^{-5} \mathrm{~W} \mathrm{~m}^{-1} \mathrm{~K}^{-1}$. The thickness of arsenene and antimonene is considered to be 3.60 and $3.83 \AA$, respectively.

\section{RESULTS AND DISCUSSION}

The buckled structure of the monolayers is illustrated in Fig. 1. For arsenene, the bond distance is $2.50 \AA$ and the

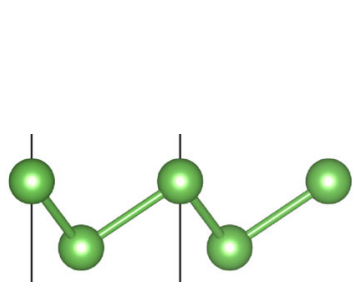

(a)

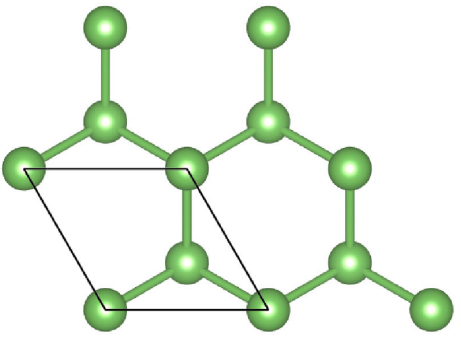

(b)

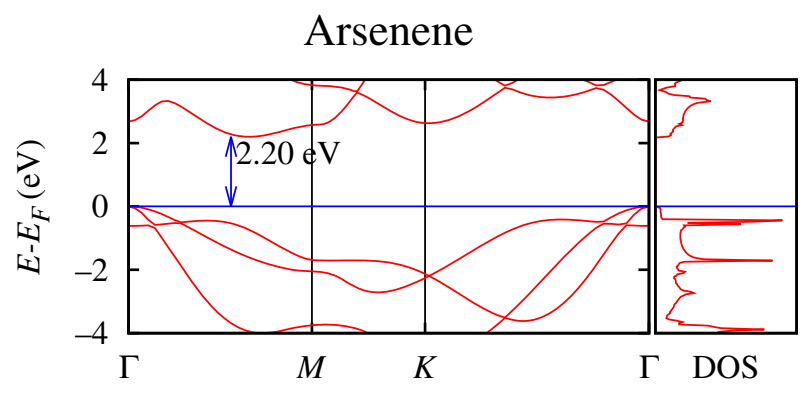

Antimonene

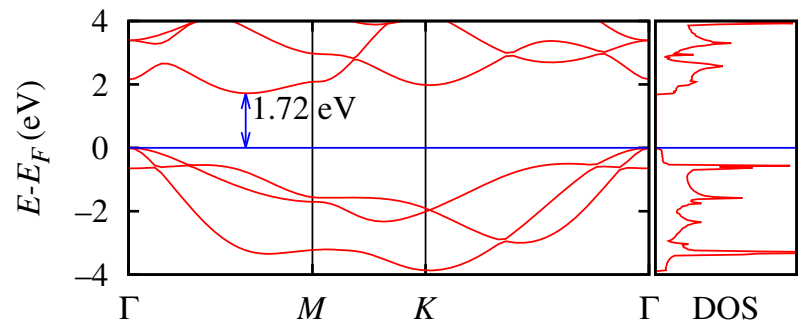

FIG. 1. Crystal structure of arsenene and antimonene viewed from (a) the side and (b) the top with corresponding HSE06 band structure and DOS.

bond angle $92.0^{\circ}$, while, for antimonene, we obtain $2.89 \AA$ and $90.9^{\circ}$, respectively. These values agree with previous studies [19,32]. The band structure and DOS are also shown in Fig. 1. The band gaps of 2.20 (arsenene) and $1.72 \mathrm{eV}$ (antimonene) exceed those of the puckered allotropes [18,19]. Figure 2 summarizes results for $S, \sigma / \tau$, and $\kappa_{e} / \tau$ (with $\tau$ being the relaxation time) as functions of the carrier concentration $(\rho)$ at different temperatures for both $n$ - and $p$-type doping. Pristine arsenene and antimonene exhibit, at $300 \mathrm{~K}$, high $S$ values of 0.30 and $0.34 \mathrm{mV} \mathrm{K}^{-1}$, respectively.

Figure 2 shows, for the two materials, a comparable thermoelectric response for $n$ - and $p$-type doping in the range of $10^{10}-10^{14} \mathrm{~cm}^{-2}$ (rigid-band approximation) and temperatures between 300 and $700 \mathrm{~K}$. The reason is that the band structures (see Fig. 1) are also very similar. We obtain for arsenene and antimonene, respectively, effective electron masses of $0.48 m_{e}$ and $0.44 m_{e}$ at the conduction-band minimum along the $\Gamma-M$ direction and effective hole masses of $0.44 m_{e}$ and $0.42 m_{e}$ at the $\Gamma$ point. The slight differences 


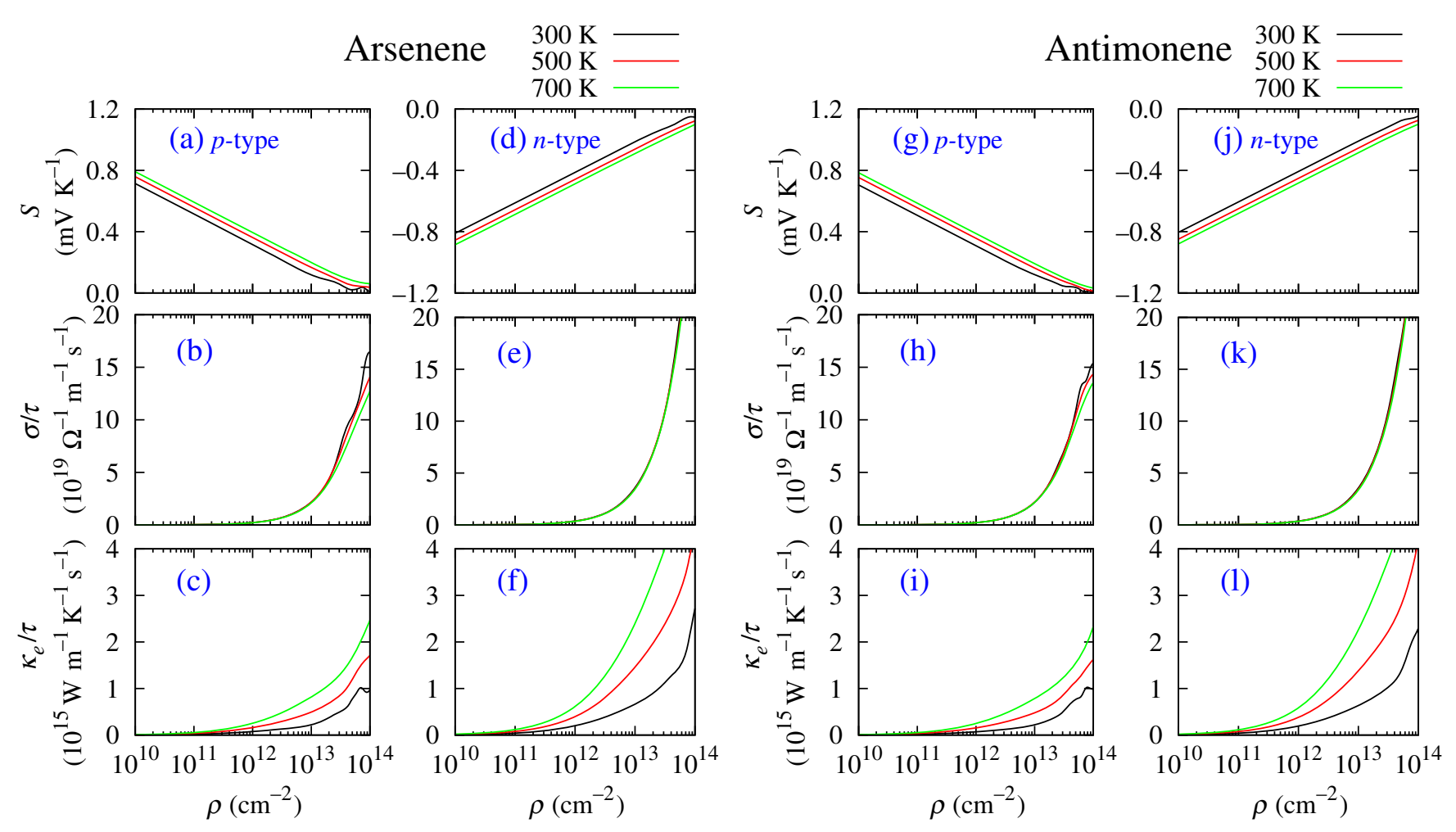

FIG. 2. Seebeck coefficient, electrical conductivity, and electronic contribution to the thermal conductivity as functions of the carrier concentration at different temperatures for both $n$ - and $p$-type doping.

in the effective masses are reflected by the results for $\sigma / \tau$ in Fig. 2. The smaller band gap of antimonene compared to arsenene also enhances $\sigma / \tau$. For both $n$ - and $p$-type doping, at a fixed temperature, the absolute value of $S$ decreases for an increasing $\rho$ and $\kappa_{e} / \tau$ grows, demonstrating that there exists an optimal $\rho$ that maximizes $Z T$. At a fixed $\rho$, an increasing temperature enhances $S$ and $\kappa_{e} / \tau$, as expected. Finally, the Wiedemann-Franz law $\left(\kappa_{e}=L \sigma T\right)$ is found to apply qualitatively.

Phonon band structures and densities of states are shown in Fig. 3. The $D_{3 d}$ point group results in $A_{1 g}$ (nondegenerate, out-of-plane) and $E_{2 g}$ (twofold-degenerate, in-plane) modes at $210 \mathrm{~cm}^{-1}\left(305 \mathrm{~cm}^{-1}\right)$ and $161 \mathrm{~cm}^{-1}\left(236 \mathrm{~cm}^{-1}\right)$ at the Brillouin zone center for arsenene (antimonene), in agreement with previous results [19]. While the higher

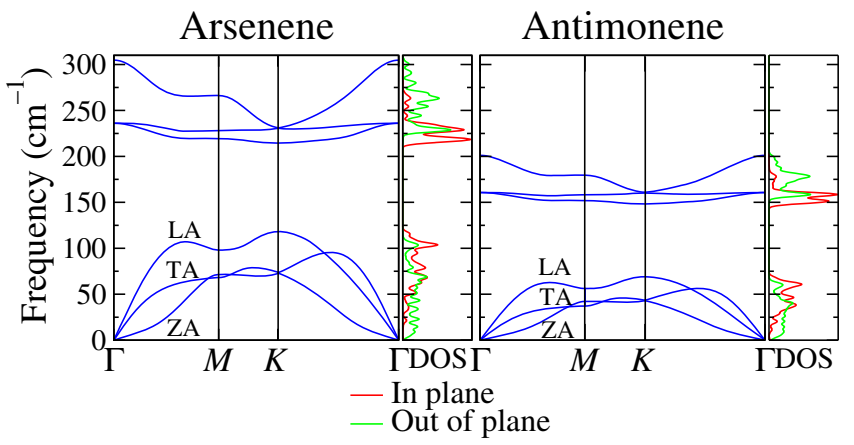

FIG. 3. Phonon dispersion and DOS. atomic mass of $\mathrm{Sb}$ (1.6 times that of As) lowers the phonon frequencies, the shape of the phonon bands remains similar. It also lowers the group velocities ( $v_{g}$, determining the flow of thermal energy), see the results calculated from the phonon band structures along the $\Gamma-M$ and $\Gamma-K$ directions in Fig. 4, and thus $\kappa_{l}$. The phonon densities of states in Fig. 3 quantify the contributions of the in-plane [longitudinal (LA), transversal (TA)] and out-of-plane [flexural (ZA)] vibrations. Importantly, the wide phonon band gaps found for both arsenene and antimonene inhibit the

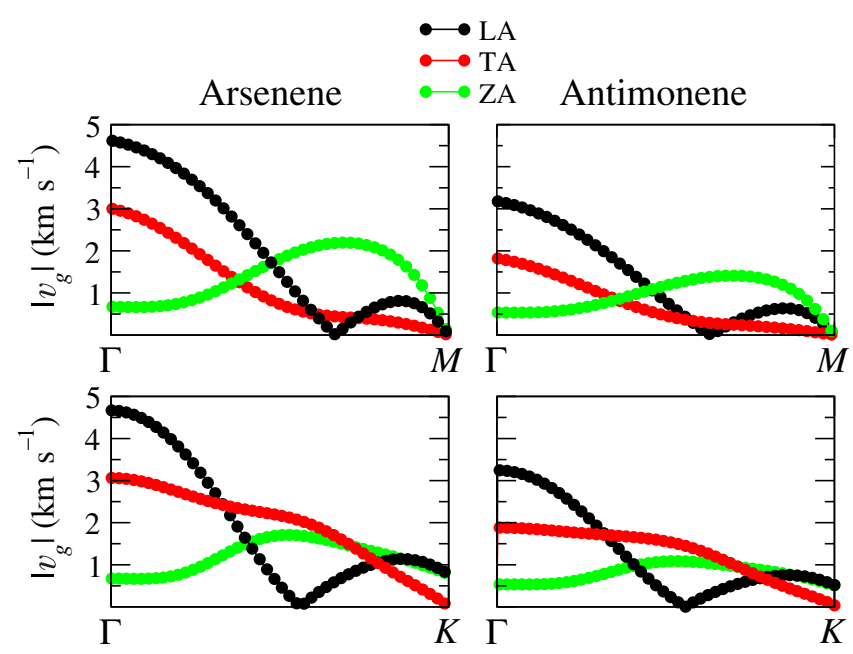

FIG. 4. Group velocity along the $\Gamma-M$ and $\Gamma-K$ directions. 


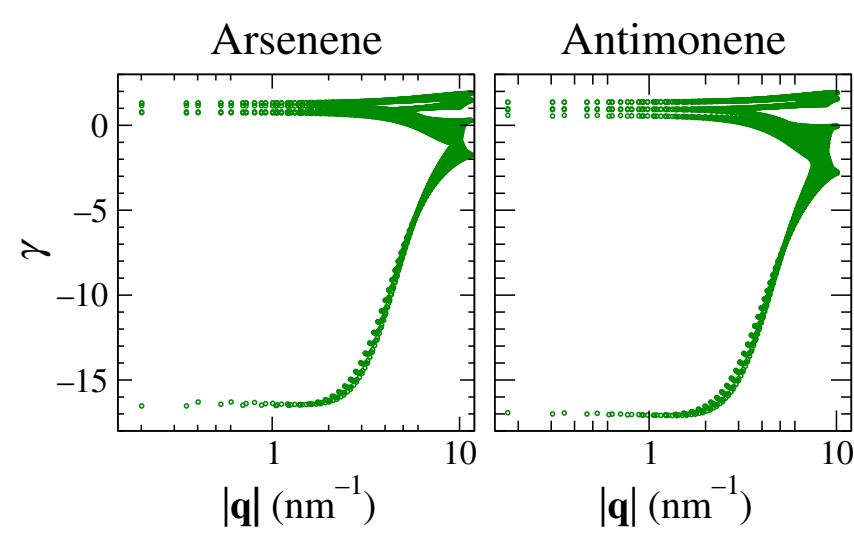

FIG. 5. Mode Grüneisen parameter.

scattering of acoustic to optical phonons, which enhances $\kappa_{l}$. We note that our calculations for $\kappa_{l}$ take into account three phonon processes (normal and Umklapp processes), as well as isotopic scattering effects. The fact that $v_{g}$ of the ZA branch is nonzero at the $\Gamma$ point (in contrast to graphene), see Fig. 4, can be attributed to the buckled structures of the materials under study [56,57].

While the in-plane and out-of-plane dielectric constants $\left(\epsilon_{\infty}\right)$ are 4.4 (1.3) and 4.9 (1.3) for arsenene (antimonene), the Born effective charges are negligible, and the longitudinal and transversal optical modes thus do not split. Similarly the ionic contributions to the dielectric constants are zero. Mode Grüneisen parameters in the irreducible Brillouin zone are shown in Fig. 5. Similar patterns for arsenene and antimonene indicate similar anharmonicity effects on $\kappa_{l}$. The total Grüneisen parameter turns out to be negative $(-0.22$ and -0.56 for arsenene and antimonene, respectively), see Fig. 5,
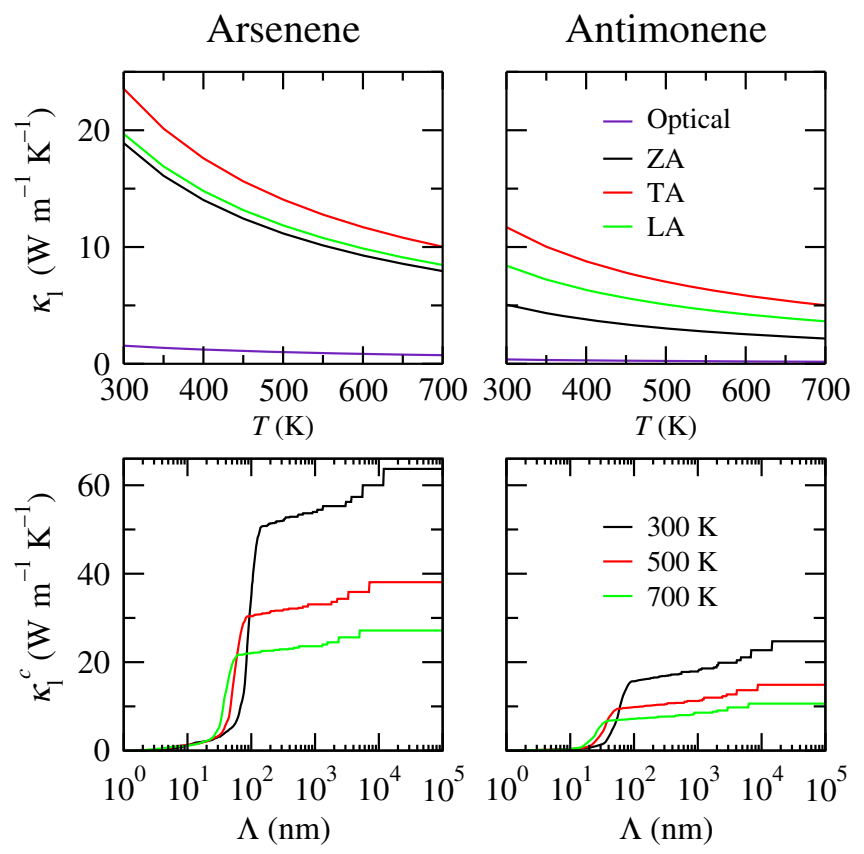

FIG. 6. In-plane lattice thermal conductivity and cumulative lattice thermal conductivity.
TABLE I. In-plane stiffness (in $\mathrm{J} \mathrm{m}^{-2}$ ), effective mass (in units of the free electron mass), and deformation-potential constant (in $\mathrm{eV}$ ) at $300 \mathrm{~K}$.

\begin{tabular}{lclcccc}
\hline \hline & $C_{2 \mathrm{D}}$ & & $m_{x}^{*}$ & $m_{y}^{*}$ & $E_{1, x}$ & $E_{1, y}$ \\
\hline Arsenene & 52 & Hole & 0.47 & 0.49 & 6.2 & 6.4 \\
& & Electron & 0.15 & 0.50 & 6.5 & 3.7 \\
Antimonene & \multirow{2}{*}{32} & Hole & 0.45 & 0.49 & 5.4 & 5.6 \\
& & Electron & 0.15 & 0.48 & 4.3 & 3.5 \\
\hline \hline
\end{tabular}

due to large contributions from the ZA vibrations, which reflects the membrane effect that is characteristic of twodimensional systems $[58,59]$. By the suppressed scattering of acoustic to optical phonons, at room temperature, the former account for about $98 \%$ of the (isotropic) in-plane $\kappa_{l}$ in both arsenene and antimonene; see Fig. 6. To determine the sample size effects, we cumulate $\kappa_{l}$ to obtain

$$
\kappa_{l}^{c}(\Lambda)=\sum_{\left|\mathbf{v}_{\lambda}\right| \tau_{\lambda}<\Lambda} \kappa_{l}\left(\mathbf{v}_{\lambda}, \tau_{\lambda}\right)
$$

where $\mathbf{v}_{\lambda}$ and $\tau_{\lambda}$ are the velocity and the relaxation time, respectively, of phonon mode $\lambda$ (see the bottom row of Fig. 6). A reduction of $\kappa_{l}^{c}$ is found when the sample dimension falls below about $100 \mathrm{~nm}$, which is more pronounced for arsenene than for antimonene.

Arsenene (hole) $\longrightarrow$ Arsenene (electron) $\square$ Antimonene (hole) $\longrightarrow$ Antimonene (electron) $\longrightarrow$
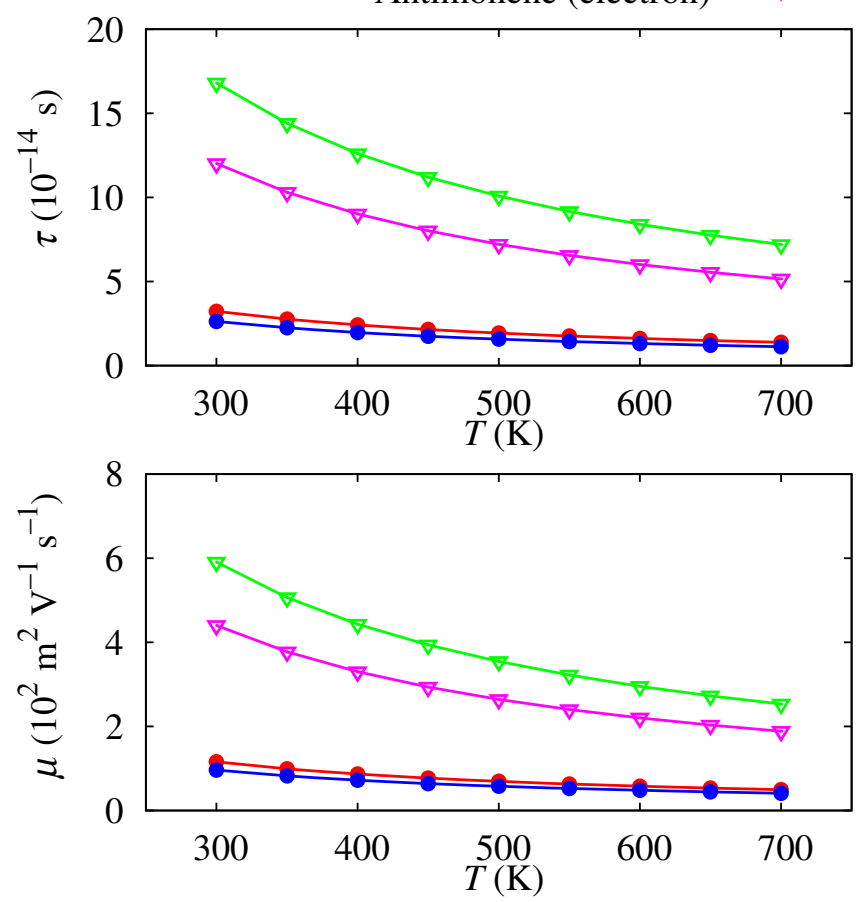

FIG. 7. Relaxation time and carrier mobility (transport along the $y$ direction) as functions of the temperature. 

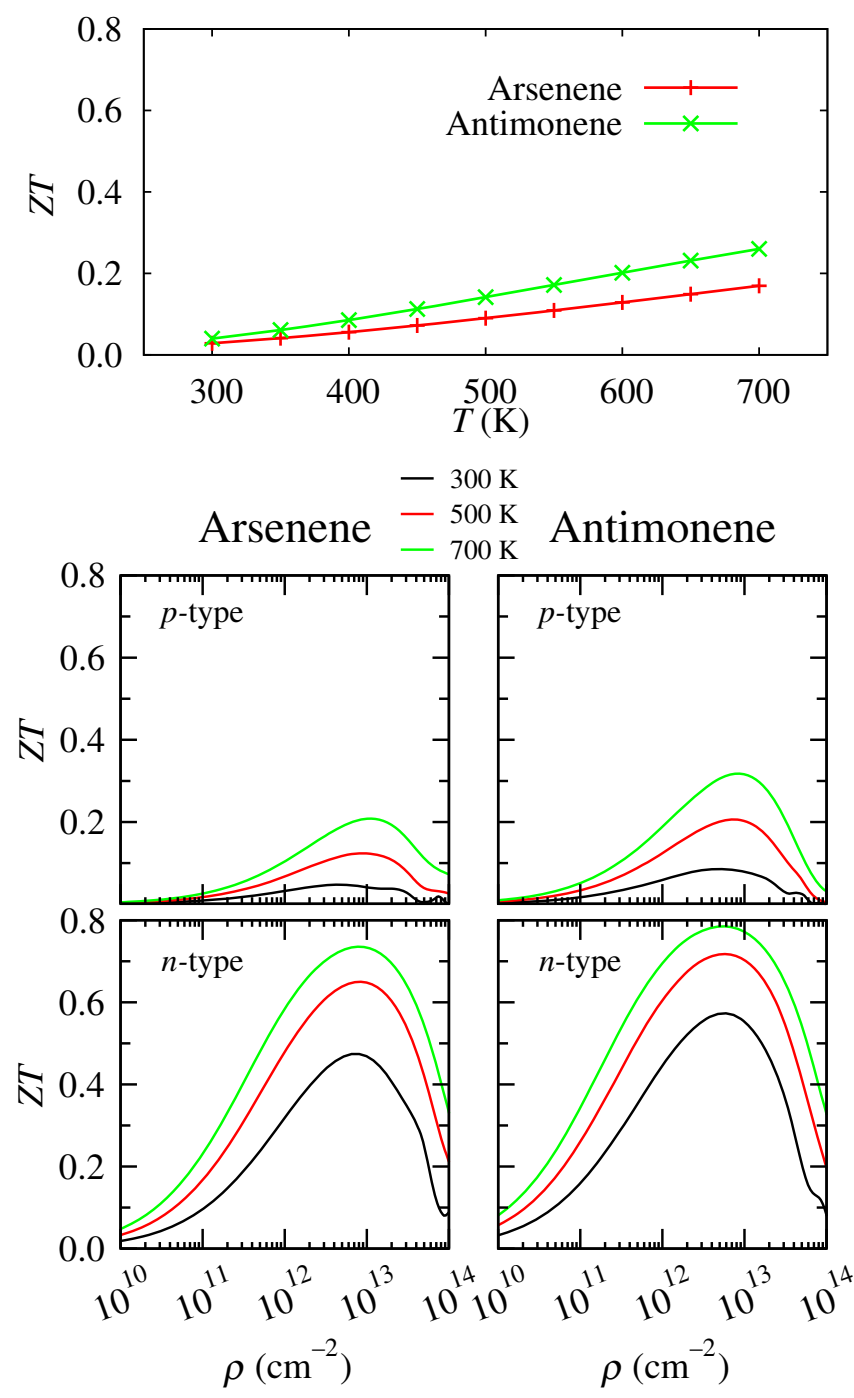

FIG. 8. Figure of merit (top panel) as a function of the temperature for the pristine materials and (bottom panels) as a function of the carrier concentration.

Within deformation-potential theory [27], we have, for transport along the $x / y$ direction,

$$
\tau_{x / y}=\frac{\hbar^{3} C_{2 \mathrm{D}}}{k_{B} T \sqrt{m_{x}^{*} m_{y}^{*}} E_{1, x / y}^{2}},
$$

where $\hbar$ is the reduced Planck's constant, $C_{2 \mathrm{D}}$ the in-plane stiffness, $k_{B}$ the Boltzmann constant, $T$ the temperature, $m_{x / y}^{*}$ the effective mass (heavy carriers in the case of degeneracy at the band edge), and $E_{1, x / y}$ the deformationpotential constant. Excellent agreement with the experiment has been found for the carrier mobility of graphene, for example, obtained by this approach [60]. The same is true for $S$ of graphene determined in the constant relaxation-time approximation [61]. Values obtained for $C_{2 \mathrm{D}}, m_{x / y}^{*}$, and $E_{1, x / y}$ are summarized in Table I. In the following, we focus on transport along the $y$ direction, as higher $Z T$ values are obtained. Both $\tau_{y}$ and the carrier mobility $\mu_{y}=\tau_{y} e / m_{y}^{*}$ are depicted in Fig. 7 as functions of the temperature, showing higher values for electrons than for holes and a decline at increasing temperatures. According to Fig. 8, we obtain ZT values of 0.17 and 0.26 , respectively, for pristine arsenene and antimonene at a temperature of $700 \mathrm{~K}$, indicating an excellent thermoelectric response. A moderate doping (a carrier concentration of approximately $10^{13} \mathrm{~cm}^{-2}$ ) enhances $Z T$ significantly for both types of carriers.

$Z T$ values approaching 0.8 upon doping make arsenene and antimonene competitors of well-established thermoelectric materials. In addition, they clearly outperform other two-dimensional materials. For example, at room temperature, more than twice the maximal $Z T$ value reported for $n$-type doped phosphorene is achieved [62]. Using Boltzmann transport theory, for graphene, a value of $Z T=0.08$ has been predicted from molecular-dynamics simulations [63] and for silicene and germanene a value of $Z T=0.4$ forms an upper limit [8] (it would be significantly reduced by a full consideration of $\kappa_{l}$ ). For monolayer transition-metal dichalcogenides, values of $Z T=0.4-1.1$ and $Z T=0.7-0.9$ have been derived based on Landauer's formalism [64] and the nonequilibrium Green's-function approach [65], respectively, whereas application of the Boltzmann transport formalism for the electrons and phonons leads to much lower values of $Z T \leq 0.13$ for $\mathrm{MoSe}_{2}$ and $\mathrm{WSe}_{2}$ [66]. Arsenene and antimonene, likewise, outperform the MXenes studied in Ref. [67].

\section{CONCLUSION}

The electronic band structures of arsenene and antimonene calculated by hybrid density-functional theory show large band gaps, which result in significant Seebeck coefficients. Because of their exceptionally low lattice thermal conductivity (which is almost purely due to the in-plane acoustical vibrations) both monolayers are characterized by an excellent thermoelectric response in comparison to other two-dimensional materials. Antimonene is slightly superior, since both the phonon frequencies and the group velocities are smaller than in arsenene. The materials are particularly promising for thermoelectric applications at room temperature, since $Z T$ values of up to 0.58 are achieved by moderate $n$-type doping of approximately $10^{13} \mathrm{~cm}^{-2}$. On the other hand, $p$-type doping turns out to be a less efficient approach.

\section{ACKNOWLEDGMENTS}

The research reported in this publication was supported by funding from King Abdullah University of Science and Technology (KAUST).

S. S. and S. K. contributed equally to this work. 


\section{APPENDIX: EFFECT OF SPIN-ORBIT COUPLING}

We check for the effect of spin-orbit coupling on the results in Fig. 2 and observe only minor modifications; see Fig. 9.

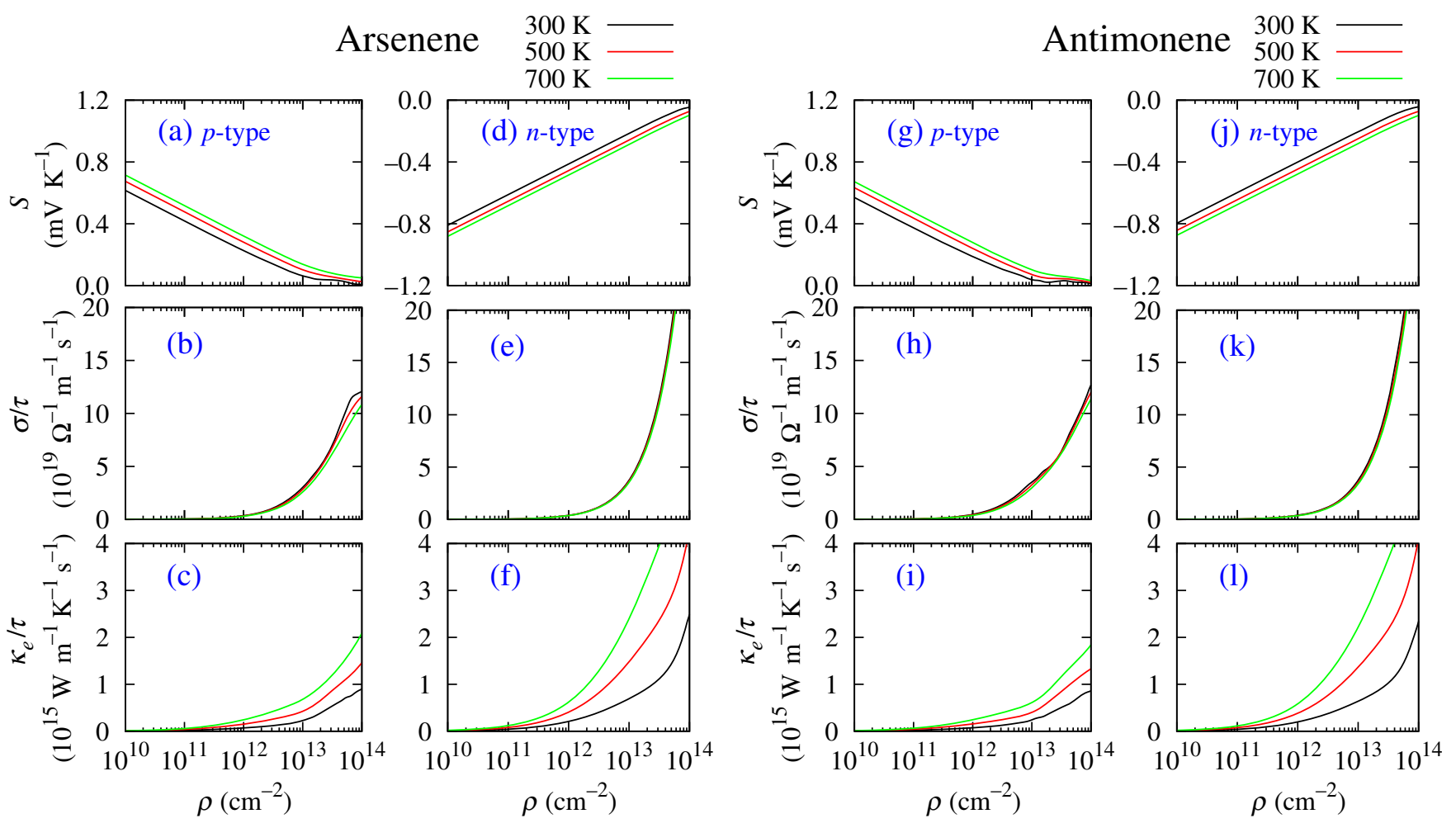

FIG. 9. Seebeck coefficient, electrical conductivity, and electronic contribution to the thermal conductivity as functions of the carrier concentration at different temperatures for both $n$ - and $p$-type doping. The spin-orbit coupling is taken into account in the calculation, in contrast to Fig. 2.

[1] F. J. DiSalvo, Thermoelectric cooling and power generation, Science 285, 703 (1999).

[2] D. M. Rowe, CRC Handbook of Thermoelectrics: Macro to Nano (CRC Press, Boca Raton, 2005).

[3] H. J. Goldsmid, Introduction to Thermoelectricity, Springer Series in Materials Science (Springer, New York, 2009).

[4] D. L. Hicks and M. S. Dresselhaus, Effect of quantum-well structures on the thermoelectric figure of merit, Phys. Rev. B 47, 12727 (1993).

[5] M. S. Dresselhaus, G. Chen, M. Y. Tang, R. Yang, H. Lee, D. Wang, Z. Ren, J.-P. Fleurial, and P. Gogna, New directions for low-dimensional thermoelectric materials, Adv. Mater. 19, 1043 (2007).

[6] G. J. Snyder and E. S. Toberer, Complex thermoelectric materials, Nat. Mater. 7, 105 (2008).

[7] Y. Xu, Z. Li, and W. Duan, Thermal and thermoelectric properties of graphene, Small 10, 2182 (2014).

[8] K. Yang, S. Cahangirov, A. Cantarero, A. Rubio, and R. D'Agosta, Thermoelectric properties of atomically thin silicene and germanene nanostructures, Phys. Rev. B 89, 125403 (2014).

[9] H. Sadeghi, S. Sangtarash, and C. J. Lambert, Enhanced thermoelectric efficiency of porous silicene nanoribbons, Sci. Rep. 5, 9514 (2015).

[10] J. Zhang, H. J. Liu, L. Cheng, J. Wei, J. H. Liang, D. D. Fan, J. Shi, X. F. Tang, and Q. J. Zhang, Phosphorene nanoribbon as a promising candidate for thermoelectric applications, Sci. Rep. 4, 6452 (2014).

[11] H. Y. Lv, W. J. Lu, D. F. Shao, and Y. P. Sun, Enhanced thermoelectric performance of phosphorene by straininduced band convergence, Phys. Rev. B 90, 085433 (2014).

[12] R. Fei, A. Faghaninia, R. Soklaski, J.-A. Yan, C. Lo, and L. Yang, Enhanced thermoelectric efficiency via orthogonal electrical and thermal conductances in phosphorene, Nano Lett. 14, 6393 (2014).

[13] L. Sun, P. H. Jiang, H. J. Liu, D. D. Fan, J. H. Liang, J. Wei, L. Cheng, J. Zhang, and J. Shi, Graphdiyne: A twodimensional thermoelectric material with high figure of merit, Carbon 90, 255 (2015). 
[14] C. Lee, J. Hong, M.-H. Whangbo, and J. H. Shim, Enhancing the thermoelectric properties of layered transition-metal dichalcogenides $2 H-M Q_{2}(M=\mathrm{Mo}, \mathrm{W} ; Q=S$, Se, Te $)$ by layer mixing: Density functional investigation, Chem. Mater. 25, 3745 (2013).

[15] W. Huang, X. Luo, C. K. Gan, S. Y. Quek, and L. Gengchiau, Theoretical study of thermoelectric properties of few-layer $\mathrm{MoS}_{2}$ and $\mathrm{WSe}_{2}$, Phys. Chem. Chem. Phys. 16, 10866 (2014).

[16] M. Khazaei, M. Arai, T. Sasaki, M. Estili, and Y. Sakka, Two-dimensional molybdenum carbides: Potential thermoelectric materials of the MXene family, Phys. Chem. Chem. Phys. 16, 7841 (2014).

[17] S. Zhang, Y. Zhong, Y. Li, Z. Chen, and H. Zeng, Atomically thin arsenene and antimonene: Semimetalsemiconductor and indirect-direct band-gap transitions, Angew. Chem., Int. Ed. Engl. 54, 3112 (2015).

[18] C. Kamal and M. Ezawa, Arsenene: Two-dimensional buckled and puckered honeycomb arsenic systems, Phys. Rev. B 91, 085423 (2015).

[19] G. Wang, R. Pandey, and S. P. Karna, Atomically thin group V elemental films: Theoretical investigations of antimonene allotropes, ACS Appl. Mater. Interfaces 7, 11490 (2015).

[20] T. Lei, C. Liu, J.-L. Zhao, J.-M. Li, Y.-P. Li, J.-O. Wang, R. Wu, H.-J. Qian, H.-Q. Wang, and K. Ibrahim, Electronic structure of antimonene grown on $\mathrm{Sb}_{2} \mathrm{Te}_{3}(111)$ and $\mathrm{Bi}_{2} \mathrm{Te}_{3}$ substrates, J. Appl. Phys. 119, 015302 (2016).

[21] H.-S. Tsai, S.-W. Wang, C.-H. Hsiao, C.-W. Chen, H. Ouyang, Y.-L. Chueh, H.-C. Kuo, and J.-H. Liang, Direct synthesis and practical band gap estimation of multilayer arsenene nanoribbons, Chem. Mater. 28, 425 (2016).

[22] D. Schiferl and C. S. Barrett, The crystal structure of arsenic at 4.2, 78, $299^{\circ} \mathrm{K}$, J. Appl. Crystallogr. 2, 30 (1969).

[23] S. Zhang, Y. Hu, H. Ziyu, B. Cai, and H. Zeng, Hydrogenated arsenenes as planar magnet and Dirac material, Appl. Phys. Lett. 107, 022102 (2015).

[24] Z. Zhu, J. Guan, and D. Tománek, Strain-induced metalsemiconductor transition in monolayers and bilayers of gray arsenic: A computational study, Phys. Rev. B 91, 161404(R) (2015).

[25] Z. Zhu, J. Guan, and D. Tománek, Structural transition in layered $\mathrm{As}_{1-x} \mathrm{P}_{x}$ compounds: A computational study, Nano Lett. 15, 6042 (2015).

[26] Z. Zhang, J. Xie, D. Yang, Y. Wang, M. Si, and D. Xue, Manifestation of unexpected semiconducting properties in few-layer orthorhombic arsenene, Appl. Phys. Express 8, 055201 (2015).

[27] Y. Wang and Y. Ding, Electronic structure and carrier mobilities of arsenene and antimonene nanoribbons: A first-principle study, Nanoscale Res. Lett. 10, 254 (2015).

[28] F. Shojael and H. S. Kang, Electronic structure and carrier mobility of two-dimensional $\alpha$ arsenic phosphide, J. Phys. Chem. C 119, 20210 (2015).

[29] Z. Y. Zhang, H. N. Cao, J. C. Zhang, Y. H. Wang, D. S. Xue, and M.S. Si, Orientation and strain modulated electronic structures in puckered arsenene nanoribbons, AIP Adv. 5, 067117 (2015).

[30] O. Ü. Aktürk, V. O. Özćelik, and S. Ciraci, Single-layer crystalline phases of antimony: Antimonenes, Phys. Rev. B 91, 235446 (2015).
[31] Y. Wang and Y. Ding, Unexpected buckled structures and tunable electronic properties in arsenic nanosheets: Insights from first-principles calculations, J. Phys. Condens. Matter 27, 225304 (2015).

[32] P. Chun-Ying, Y. Xiao-Tao, J. Hua-Long, F.-W. Zhang, Z.-W. Lu, J.-B. He, and D.-W. Zhou, Two-dimensional arsenic monolayer sheet predicted from first-principles, Chin. Phys. B 24, 036301 (2015).

[33] M. Zeraati, S. M. V. Allaei, A. I. Sarsari, M. Pourfath, and D. Donadio, Highly anisotropic thermal conductivity of arsenene: An ab initio study, Phys. Rev. B 93, 085424 (2016).

[34] S. Wang, W. Wang, and G. Zhao, Thermal transport properties of antimonene: An ab initio study, Phys. Chem. Chem. Phys. 18, 31217 (2016).

[35] T. Zhang, Y.-Y. Qi, X.-R. Chen, and L.-C. Cai, Predicted low thermal conductivities in antimony films and the role of chemical functionalization, Phys. Chem. Chem. Phys. 18, 30061 (2016).

[36] S. K. Gupta, Y. Sonvane, G. Wang, and R. Pandey, Size and edge roughness effects on thermal conductivity of pristine antimonene allotropes, Chem. Phys. Lett. 641, 169 (2015).

[37] B. Peng, D. Zhang, H. Zhang, H. Shao, G. Ni, Y. Zhu, and $\mathrm{H}$. Zhu, The conflicting role of buckled structure in phonon transport of 2D group-IV and group-V materials, Nanoscale 9, 7397 (2017).

[38] Y. M. Zuev, W. Chang, and P. Kim, Thermoelectric and Magnetothermoelectric Transport Measurements of Graphene, Phys. Rev. Lett. 102, 096807 (2009).

[39] P. Wei, W. Bao, Y. Pu, C. N. Lau, and J. Shi, Anomalous Thermoelectric Transport of Dirac Particles in Graphene, Phys. Rev. Lett. 102, 166808 (2009).

[40] S.-G. Nam, D.-K. Ki, and H.-J. Lee, Thermoelectric transport of massive Dirac fermions in bilayer graphene, Phys. Rev. B 82, 245416 (2010).

[41] X. Wu, Y. Hu, M. Ruan, N. K. Madiomanana, C. Berger, and W. A. de Heer, Thermoelectric effect in high mobility single layer epitaxial graphene, Appl. Phys. Lett. 99, 133102 (2011).

[42] N. Xiao, X. Dong, L. Song, D. Liu, Y. Tay, S. Wu, L.-J. Li, Y. Zhao, T. Yu, H. Zhang, W. Huang, H. H. Hng, P. M. Ajayan, and Q. Yan, Enhanced thermopower of graphene films with oxygen plasma treatment, ACS Nano 5, 2749 (2011).

[43] A. N. Sidorov, A. Sherehiy, R. Jayasinghe, R. Stallard, D. K. Benjamin, Q. Yu, Z. Liu, W. Wu, H. Cao, Y. P. Chen, Z. Jiang, and G. U. Sumanasekera, Thermoelectric power of graphene as surface charge doping indicator, Appl. Phys. Lett. 99, 013115 (2011).

[44] Y. Anno, Y. Imakita, K. Takei, S. Akita, and T. Arie, Enhancement of graphene thermoelectric performance through defect engineering, 2D Mater. 4, 025019 (2017).

[45] J. Wu, H. Schmidt, K. K. Amara, X. Xu, G. Eda, and B. Özyilmaz, Large thermoelectricity via variable range hopping in chemical vapor deposition grown single-layer $\mathrm{MoS}_{2}$, Nano Lett. 14, 2730 (2014).

[46] J. Pu, K. Kanahashi, N. T. Cuong, C.-H. Chen, L.-L. Li, S. Okada, H. Ohta, and T. Takenobu, Enhanced thermoelectric power in two-dimensional transition metal dichalcogenide monolayers, Phys. Rev. B 94, 014312 (2016). 
[47] G. Kresse and D. Joubert, From ultrasoft pseudopotentials to the projector augmented-wave method, Phys. Rev. B 59, 1758 (1999).

[48] The atomic forces are converged within $0.01 \mathrm{eV}^{-1}$ and the total energy within $10^{-6} \mathrm{eV}$. These and all other technical parameters are chosen such that the associated errors in the evaluated quantities are limited to about $1 \%$.

[49] G. K. H. Madsen and D. J. Singh, BoltzTraP: A code for calculating band-structure dependent quantities, Comput. Phys. Commun. 175, 67 (2006).

[50] J. Heyd and G. E. Scuseria, Efficient hybrid density functional calculations in solids: Assessment of the HeydScuseria-Ernzerhof screened Coulomb hybrid functional, J. Chem. Phys. 121, 1187 (2004).

[51] W. Li, L. Lindsay, D. A. Broido, D. A. Stewart, and N. Mingo, Thermal conductivity of bulk and nanowire $\mathrm{Mg}_{2} \mathrm{Si}_{x} \mathrm{Sn}_{1-x}$ alloys from first principles, Phys. Rev. B 86, 174307 (2012).

[52] W. Li, J. Carrete, N. A. Katcho, and N. Mingo, SHEngBTE: A solver of the Boltzmann transport equation for phonons, Comput. Phys. Commun. 185, 1747 (2014).

[53] M. Wagner-Reetz, D. Kasinathan, W. Schnelle, R. CardosoGil, H. Rosner, Y. Grin, and P. Gille, Phonon-drag effect in $\mathrm{FeGa}_{3}$, Phys. Rev. B 90, 195206 (2014).

[54] A. Togo, F. Oba, and I. Tanaka, First-principles calculations of the ferroelastic transition between rutile-type and $\mathrm{CaCl}_{2}$ type $\mathrm{SiO}_{2}$ at high pressures, Phys. Rev. B 78, 134106 (2008).

[55] A. Togo, SpgLiB: A C library for finding and handling crystal symmetries, https://atztogo.github.io/spglib/.

[56] B. Peng, H. Zhang, H. Shao, Y. Xu, X. Zhang, and H. Zhu, Low lattice thermal conductivity of stanene, Sci. Rep. 6, 20225 (2016).
[57] L.-F. Huang, P.-L. Gong, and Z. Zeng, Phonon properties, thermal expansion, and thermomechanics of silicene and germanene, Phys. Rev. B 91, 205433 (2015).

[58] I. M. Lifshitz, Thermal properties of chain and layered structures at low temperatures, Zh. Eksp. Teor. Fiz. 22, 475 (1952).

[59] N. A. Abdullaev, Grüneisen parameters for layered crystals, Phys. Solid State 43, 727 (2001).

[60] M.-Q. Long, L. Tang, D. Wang, L. Wang, and Z. Shuai, Theoretical predictions of size-dependent carrier mobility and polarity in graphene, J. Am. Chem. Soc. 131, 17728 (2009).

[61] R. D'Souza and S. Mukherjee, First-principles study of the electrical and lattice thermal transport in monolayer and bilayer graphene, Phys. Rev. B 95, 085435 (2017).

[62] H. Y. Lv, W. J. Lu, D. F. Shao, and Y. P. Sun, Large thermoelectric power factors in black phosphorus and phosphorene, arXiv:1404.5171.

[63] A. H. Reshak, S. A. Khan, and S. Auluck, Thermoelectric properties of a single graphene sheet and its derivatives, J. Mater. Chem. C 2, 2346 (2014).

[64] D. Wickramaratne, F. Zahid, and R. K. Lake, Electronic and thermoelectric properties of few-layer transition metal dichalcogenides, J. Chem. Phys. 140, 124710 (2014).

[65] K.-X. Chen, X.-M. Wang, D.-C. Mo, and S.-S. Lyu, Thermoelectric properties of transition metal dichalcogenides: From monolayers to nanotubes, J. Phys. Chem. C 119, 26706 (2015).

[66] S. Kumar and U. Schwingenschlögl, Thermoelectric response of bulk and monolayer $\mathrm{MoSe}_{2}$ and $\mathrm{WSe}_{2}$, Chem. Mater. 27, 1278 (2015).

[67] A. N. Gandi, H. N. Alshareef, and U. Schwingenschlögl, Thermoelectric performance of the MXenes $M_{2} \mathrm{CO}_{2}$ ( $M=\mathrm{Ti}$, Zr, or Hf), Chem. Mater. 28, 1647 (2016). 